\title{
Epidural Analgesia and Vaginal Delivery in a Patient with Aortic Stenosis and Insufficiency
}

\author{
Engin Erturk Habib Bostan Ahmet Eroglu \\ Department of Anesthesiology and Intensive Care, Faculty of Medicine, Karadeniz Technical University, \\ Trabzon, Turkey
}

\section{Key Words}

Epidural analgesia • Vaginal delivery · Aortic stenosis •

Aortic insufficiency $\cdot$ Ropivacaine

\begin{abstract}
Objective: To report a successful vaginal delivery using slow infusion of epidural analgesia in a patient with both severe aortic stenosis and insufficiency. Clinical Presentation and Intervention: A 26-year-old primigravid patient presented to our hospital for delivery. She had aortic stenosis and insufficiency due to rheumatic fever. Although the obstetrician recommended cesarean section owing to her cardiac status, she insisted upon vaginal delivery. We performed low-dose epidural analgesia with $10 \mathrm{ml}$ of $0.125 \%$ ropivacaine and $20 \mu \mathrm{g}$ fentanyl. She had spontaneous vaginal delivery without complication. Conclusion: This case showed that in spite of the cardiac pathology, vaginal delivery under low-dose slow infusion of epidural analgesia was successful and therefore may be a safe alternative to cesarean section for cardiac patients.

Copyright $\odot 2011$ S. Karger AG, Basel
\end{abstract}

\section{Introduction}

Cardiovascular disease in pregnancy is the most common cause of nonobstetric maternal mortality [1]. Substantial changes, such as increases in cardiac output (40-50\%) and blood volume (40\%), occur in the cardiovascular system during pregnancy, whereas systemic peripheral resistance and arterial pressure decrease [2]. These changes may cause left ventricular outflow tract obstruction [3]. In pregnancy with both aortic stenosis and insufficiency, left ventricular outflow is more limited. Fixed left ventricular outflow tract obstruction causes a restriction of stroke volume and cardiac output in pregnancy. In addition, anxiety, exertion and the pain of labor and delivery increase the hemodynamic stress on the cardiovascular system and may trigger cardiovascular decompensation. Pregnant women with both aortic stenosis and insufficiency are therefore at increased risk of cardiovascular complications, including heart failure, cardiac collapse and death. Aortic insufficiency is well tolerated in pregnancy, but aortic stenosis may become symptomatic and clinically significant [4]. Cardiac output depends entirely on heart rate and preload in aortic stenosis. In other words, cardiac output cannot be increased by the usual physiologic mechanisms of greater stroke volume, because the effective aortic valve area is smaller than nor-

\section{KARGER \\ Fax +4161306 1234 \\ E-Mail karger@karger.ch}

www.karger.com
C 2011 S. Karger AG, Basel

1011-7571/11/0206-0574\$38.00/0

Accessible online at:

www.karger.com/mpp
Dr. Engin Erturk

Department of Anesthesiology and Intensive Care

Faculty of Medicine, Karadeniz Technical University

Trabzon (Turkey)

Tel. +90 462377 5898, E-Mail engin_md@yahoo.com 
mal. Therefore, both tachycardia and bradycardia must be avoided, and adequate preload must be maintained during labor in such patients. In addition, inferior vena caval compression and prolonged efforts at pushing in the second stage of labor may occlude venous return and reduce preload and cardiac output.

Aortic insufficiency is rarely symptomatic in pregnant patients. Chronic volume overload of the left ventricle results in hypertrophy and dilatation. Pain, with resultant increases in vascular resistance and bradycardia that will increase the time for regurgitant flow, must be avoided in aortic insufficiency during labor. Since vaginal delivery may cause undesirable effects of pain and Valsalva maneuver, cesarean section is usually recommended in such cases. We report a case of successful vaginal delivery in a cardiac patient.

\section{Case Report}

A 26-year-old primigravid patient with severe aortic stenosis and aortic insufficiency was admitted to the Obstetric Service. She had suffered rheumatic fever at 6 years of age, with aortic valve disease being diagnosed the following year. She had no history of medication other than endocarditis prophylaxis with depot penicillin.

The patient had a history of weakness, dizziness and shortness of breath, especially increased in frequency after 30 weeks of gestation. Her last menstruation was 39 weeks and 6 days prior, so that the expected date of delivery would be 2 days hence. She experienced 2 syncopal episodes in the last trimester. Cardiologic examination revealed paroxysmal sinus tachycardia up to 130 beats/min, and palpitations precipitated by walking or climbing stairs. Electrocardiogram demonstrated left ventricular hypertrophy (fig. 1). A transthoracic echocardiogram demonstrated severe left ventricular dilatation and hypertrophy, diastolic interventricular septum diameter of $16 \mathrm{~mm}$, left ventricular end-diastolic diameter of $55 \mathrm{~mm}$, aortic valve area of $0.8 \mathrm{~cm}^{2}$, mean aortic gradient of $48 \mathrm{~mm} \mathrm{Hg}$, and left ventricular ejection fraction of $55 \%$. Arterial blood pressure (BP) was $125 / 50 \mathrm{~mm} \mathrm{Hg}$ and heart rate was 92 beats/min.

Initially, the obstetrician recommended cesarean section in order to avoid cardiovascular decompensation by sympathetic activation during vaginal delivery, but the patient preferred vaginal delivery if possible. Therefore, low-dose epidural analgesia was planned after consultation with the cardiologist, obstetrician and patient. The delivery room was prepared for the patient. Required equipment (defibrillation, central venous and pulmonary arterial catheterization, invasive arterial monitorization, intubation, resuscitation, mechanical ventilation facilities) was provided.

The patient had irregular contractions, and was admitted to the delivery room at 40 weeks +2 days of gestation. Low-dose oxytocin (1-2 $\mathrm{mIU} / \mathrm{min}$ ) infusion was administered to the patient for induction. Electrocardiography, pulse oximetry and invasive radial arterial BP monitoring were established. An epidural catheter was placed uneventfully at the $\mathrm{L}_{3-4}$ lumbar interspace while the patient was in the left lateral position. At $4 \mathrm{~cm}$ cervical dilatation, $10 \mathrm{ml}$ of

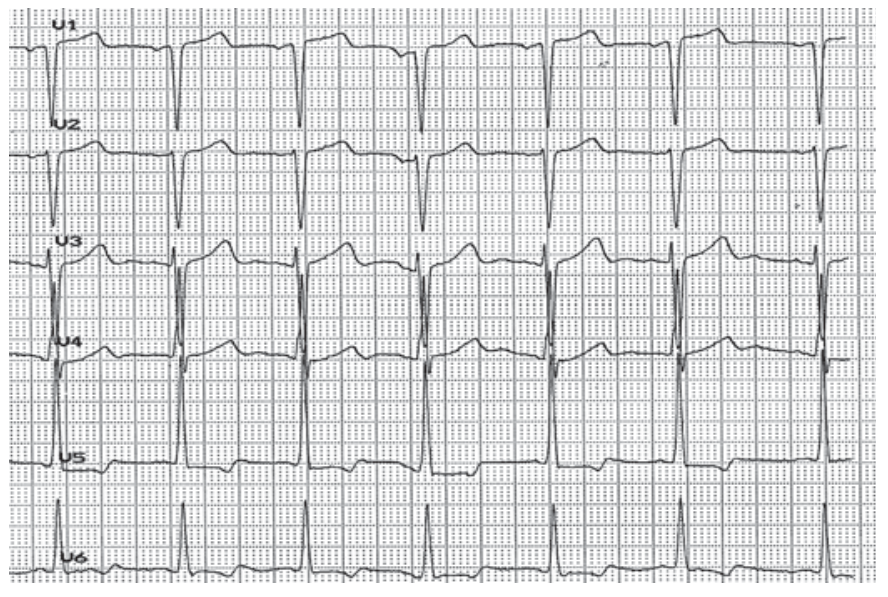

Fig. 1. Left ventricular hypertrophy.

$0.125 \%$ ropivacaine and $20 \mu \mathrm{g}$ fentanyl were administered via an epidural catheter over $10 \mathrm{~min}$, and a further $500 \mathrm{ml}$ of isotonic saline was then given intravenously over $30 \mathrm{~min}$ to prevent likely hypotension due to epidural block. Hypotension was considered to be systolic $\mathrm{BP}<80 \mathrm{~mm} \mathrm{Hg}$. In case of the development of hypotension, ephedrine treatment was planned with incremental 5-mg doses.

Adequate analgesia was established at $\mathrm{T}_{10}$ dermatome without motor block. One hour later, an infusion of $0.125 \%$ ropivacaine with $1 \mu \mathrm{g} / \mathrm{ml}$ of fentanyl was initiated at $10 \mathrm{ml} / \mathrm{h}$ and titrated between 8 and $12 \mathrm{ml} / \mathrm{h}$ as labor progressed. The patient remained comfortable with a heart rate of $70-95$ beats/min, systolic BP $>100$ $\mathrm{mm} \mathrm{Hg}$ and oxygen saturation $>97 \%$ throughout labor. She was not allowed prolonged efforts to push in the second stage to prevent occluding venous return and reducing preload and cardiac output. After $5 \mathrm{~h}$, the patient reached complete cervical dilatation. The second stage of labor lasted $25 \mathrm{~min}$ and then a 3,200-gram boy, with Apgar scores of 8 and 10 at 1 and 5 min, respectively, was delivered without any obstetric intervention. Ten international units of oxytocin were administered as an infusion in $500 \mathrm{ml}$ of isotonic saline over $1 \mathrm{~h}$. The placenta was delivered spontaneously 20 min after delivery. Hypotension did not occur during labor and ephedrine was not needed. Breast feeding was started spontaneously without any problem. The patient was observed closely for 2 days during the postpartum period and then discharged with suggestions about contraception.

\section{Discussion}

Maternal mortality may be increased in cases with severe aortic valve disease [5]. Cesarean section is usually performed for cardiac patients to avoid undesirable effects of pain and the marked Valsalva effect of prolonged efforts at pushing in the second stage $[6,7]$.

The use of neuroaxial block in patients with clinically significant cardiac disease is a controversial issue because 
of possible cardiovascular fluctuations with blockade of the autonomic system $[8,9]$. Traditionally, epidural anesthesia or analgesia is considered to be contraindicated in cardiovascular disease because of excessive peripheral vasodilatation and hypotension with a fixed cardiac output. Hypotension in these patients can theoretically produce myocardial ischemia and tachycardia leading to increasing myocardial oxygen consumption and reducing left ventricular filling time. However, there are differing opinions regarding the safest management of labor and delivery for such patients.

Since epidural analgesia reduces catecholamine release and hemodynamic stress, it has frequently been used of late in diluted forms for vaginal delivery in these patients. Suntharalingam et al. [3] used the low-dose technique with a $0.1 \%$ bupivacaine and fentanyl combination in their 5 -case series. They concluded that epidural analgesia may be used for delivery in aortic stenosis with close invasive monitoring. We also used $0.125 \%$ ropicaine and fentanyl as an epidural infusion and thus encountered no hypotension or tachycardia. It is suggested that epidural infusion rather than bolus doses be used in order to avoid epidural block-associated cardiovascular side effects.

The selection of a local anesthetic and dosage thereof is important in neuroaxial block for cardiac patients. Relatively high doses of a local anesthetic may cause severe cardiovascular complications in these patients. Bupivacaine, which is frequently used in epidural anesthesia or analgesia, is a potent local anesthetic, high doses of which may cause severe hemodynamic side effects in cardiac patients. However, ropivacaine is better tolerated than bupivacaine in patients with cardiovascular disease. Knudsen et al. [10] stated that ropivacaine was less neurotoxic and cardiotoxic than bupivacaine. We therefore used ropivacaine in diluted form for our patient. On the other hand, ropivacaine causes less motor block than bupivacaine or other local anesthetics. This property of ropivacaine provides a great advantage in allowing sufficient effort for delivery. In the presence of motor block, the second stage lasts longer than estimated.

Oxytocin is frequently used to enhance postpartum uterine contraction. However, the administration of an intravenous oxytocin bolus has resulted in severe hypotension and tachycardia due to its direct relaxing effect on vascular smooth muscle [9]. These changes can be catastrophic in the presence of both aortic stenosis and insufficiency. Thus, if oxytocin administration is required, it must not be administered as an intravenous bolus to avoid these likely catastrophic effects. We administered $10 \mathrm{IU}$ of oxytocin as an infusion over $1 \mathrm{~h}$, and encountered no side effect resulting from it.

Generally, primigravid patients experience a $25-40 \%$ cesarean section rate depending on the institution. In such a case, we planned epidural anesthesia with further $15 \mathrm{ml}$ of $0.125 \%$ ropivacaine epidural bolus injection for cesarean section, if there was time to perform the blockade. In case of a blockade failure or emergency cesarean section, general anesthesia was planned and discussed with the patient in advance to cover such contingencies. A management plan to correct her severe disease prior to further pregnancies was made, including close anesthetic, cardiologic and gynecologic evaluation.

\section{Conclusion}

This case showed that in spite of the cardiac pathology, vaginal delivery under low-dose slow infusion of epidural analgesia was successful and therefore may be a safe alternative to cesarean section for cardiac patients.

\section{References}

1 Moghbeli N, Pare E, Webb G: Practical assessment of maternal cardiovascular risk in pregnancy. Congenit Heart Dis 2008;3:308-316.

$>2$ Hess W: Cardiovascular diseases during pregnancy. Considerations for the anesthesiologist. Anaesthesist 1995;44:395-404.

$>3$ Suntharalingam G, Dob D, Yentis SM: Obstetric epidural analgesia in aortic stenosis: a low-dose technique for labour and instrumental delivery. Int J Obstet Anesth 2001;10: 129-134.

4 Gomar C, Errando CL: Neuroaxial anaesthesia in obstetrical patients with cardiac disease. Curr Opin Anaesthesiol 2005;18:507-512.
5 Arias F, Pineda J: Aortic stenosis and pregnancy. J Reprod Med 1978;20:229-232.

$\checkmark 6$ Xia VW, Messerlian AK, Mackley J, Calmes SH, Matevosian R: Successful epidural anesthesia for cesarean section in a parturient with severe aortic stenosis and a recent history of pulmonary edema - A case report. J Clin Anesth 2006;18:142-144.

7 Dua S, Maurtua MA, Cywinski JB, Deogaonkar A, Waters JH, Dolak JA: Anesthetic management for emergency cesarean section in a patient with severe valvular disease and preeclampsia. Int J Obstet Anesth 2006;15: 250-253.
8 Whitfield A, Holdcroft A: Anaesthesia for caesarean section in patients with aortic stenosis: the case for general anaesthesia. Anaesthesia 1998;53:109-112.

9 Brighouse D: Anaesthesia for caesarean section in patients with aortic stenosis: the case for regional anaesthesia. Anaesthesia 1998; 53:107-109.

10 Knudsen K, Beckman Suurküla M, Blomberg S, Sjövall J, Edvardsson N: Central nervous and cardiovascular effects of iv infusions of ropivacaine, bupivacaine and placebo in volunteers. Br J Anaesth 1997;78: 507-514 